\title{
Temporal perturbations cause global sensorimotor adaptation
}

\author{
Nadine Schlichting*1 ${ }^{1}$, Tatiana Kartashova ${ }^{1}$, Michael Wiesing ${ }^{1}$, Eckart Zimmermann ${ }^{1}$ \\ ${ }^{1}$ Institute for Experimental Psychology \\ Heinrich-Heine-University Düsseldorf, Germany \\ *corresponding author
}

\section{Correspondence}

Nadine Schlichting

nadine.schlichting@hhu.de

\section{Competing Interests}

The authors declare no competing interests.

\section{Funding}

This research was supported by the European Research Council (project moreSense, grant agreement 757184) and by the Deutsche Forschungsgemeinschaft (DFG, ZI 1456). 


\begin{abstract}
Complex movements require the fine-tuned temporal interplay of several effectors. If the temporal properties of one of these effectors were distorted, all other movement plans would need to be recalibrated in order to produce successful and well-timed behavior. Here, we tested whether timecritical and goal-oriented movements are affected more globally by adapting to a temporal perturbation compared to the beknown direction, movement type and context specificity of spatial adaptation. In a ready-set-go paradigm, participants reproduced the interval between ready- and set-signals by performing different arm and hand movements in Virtual Reality (VR). Halfway through the experiments we introduced a temporal perturbation, such that movements in VR were artificially slowed down. Participants had to adapt their behavior (i.e., speed up their movements) to sustain performance. In three experiments, we found that adaptation effects were not affected by, but transferred to different movement types, interval ranges, target locations, and environmental contexts. Thus, unlike recalibrating to spatial perturbations, the temporal planning of motor actions is recalibrated globally within the motor system, allowing smooth coordination of behavior in time.
\end{abstract}

Keywords: interval timing, visuomotor adaptation, motor planning, VR 


\section{Temporal perturbations cause global sensorimotor adaptation}

In a $100 \mathrm{~m}$ sprint race, a good start can make the difference in winning the gold or silver medal. One crucial aspect to nail the start is to measure the interval between the ready-and setsignal in order to predict when the go-signal will occur, so that the athlete can immediately leave the starting block and, ideally, save precious milliseconds. In other words, the athlete needs to make accurate and precise temporal estimates in order to produce well-timed behavior. This requirement is fulfilled best if these estimates are directly entailed in the motor planning of the sprint start movements, since transfer between representations might induce noise and delays (see also Remington et al. ${ }^{1}$ ). Indeed, Jazayeri and Shadlen ${ }^{2}$ demonstrated in macaques that in intra-parietal cortex temporal intervals are measured prospectively in relation to the desired motor plan to reproduce these intervals. Many movements, like a sprint start, reaching, or grasping require the concerted interplay of several effectors and movement types - any specificity of temporal estimates for effectors or movement types would, again, introduce neural noise and affect performance. Thus, time and timing may be a global property of the motor system, rather than being linked to specific motor actions or even being an additional input from a clock-system ${ }^{3-5}$.

If temporal estimates are entailed in motor planning and actions, then motor planning and actions may alter our ability to measure time conversely. Indeed, evidence for the influence of motor actions on duration estimates is accumulating: The frequency of finger tapping ${ }^{6-8}$; the length ${ }^{9}$ or type ${ }^{10}$ of movements; or the mere preparation of a ballistic action ${ }^{11}$ bias temporal estimates. In these studies, the motor action is task-irrelevant and experimental manipulations depend on the participant's ability to consciously alter specific movement parameters. Hence, it is impossible to say whether there is a clock-system informing the motor system and vice versa, or whether they are essentially the same. Interval production and reproduction paradigms in which, just like in the sprint example, a time-critical motor action (e.g., a saccade, button press, or arm movement) is an essential part the task, offer additional insights into the entanglement of the motor system and time. One such insight is that the in interval reproduction tasks well-known temporal context effect, in which duration estimates are biased towards previously perceived intervals (for a review, see Van Rijn ${ }^{12}$ ), has been shown to originate in motor areas of the brain ${ }^{2,13}$ and occur already during the encoding of the to-be-reproduced interval, during which no motor action is executed ${ }^{14,15}$ and decision biases can be ruled out ${ }^{16}$. Thus, as proposed in the previous paragraph, there may be no clock input to the motor system, because time is inherently rooted within it.

While the interval timing field becomes more and more interested in motor-aspects occurring in timing tasks (see above for examples), focusing on temporal aspects in well-known sensory-motor or motor learning paradigms is scarcer (but see ${ }^{17-22}$ ). One such paradigm is sensorimotor adaptation (for a review, see Krakauer et al. ${ }^{23}$ or Panico et al. ${ }^{24}$ ): Because of a perturbation in the environment (e.g., by wearing prism goggles that displace the visual field, or by moving a cursor that is subjected to a predictable force field), participants need to adapt their movement plans in order to successfully reach a target. Upon removal of the perturbation, adapted behavior sustains until participants de-adapt (i.e., re-adapt to normal conditions). The persistence of aftereffects is commonly interpreted as proof for an implicit updating of motor plans based on execution details (e.g., spatiotemporal coordination of limbs) and predicted outcome, causing 
adaptation effects ${ }^{23}$. Models explaining sensorimotor adaptation effects generally assume that the sensorimotor system tracks errors over the history of trials and uses these errors to adapt and guide subsequent movements in a closed-loop and feed-forward-fashion ${ }^{25,26}$. Sensorimotor adaptation to artificial spatial errors are generally direction specific, and other transfer effects (e.g., transfer to another movement or limb) seem to be highly dependent on task details (e.g., effector-transfer can be observed if the movements target the same goal ${ }^{27}$; task-transfer can be observed if the movement goal is goal-directed and occurs in the same environment ${ }^{21}$ ). Similar non-transferability has been observed in spatiotemporal adaptation paradigms, in which a delay perturbation is learned primarily through spatial feedback. That is, spatial and spatiotemporal adaptation is not causing a global adaptation of the motor system. In contrast, and given the idea of a more global representation of time within the motor system, adaptation to a temporal perturbation without spatial influence should affect all motor actions, regardless of movement type, target, or environment.

In the current study, we tested the transferability of temporal adaptation effects. We asked participants to reproduce intervals by performing different time-critical actions. The temporal estimate was directly contingent on the movement dynamics, and the error following a movement would inform about its temporal accuracy. The study was conducted in Virtual Reality (VR), enabling us to provide systematically distorted feedback about the movement and its temporal accuracy that leads to temporal adaptation of the movement. In a ready-set-go paradigm, the interval between ready and set had to be reproduced by performing either a ballistic finger movement (clicking a controller button, clicking reproduction) or by a continuous movement of the arm to hit a target at the time of the go-signal (pointing reproduction; note that contrary to the sprint example, the movement had to be completed by the time of the go-signal, see Figure 1A). During the adaptation phase, feedback about pointing performance was manipulated such that movements were artificially slowed down, forcing participants to adapt their movement speed in order to sustain performance. In three experiments, we tested whether adaptation to a temporal perturbation is specific to the movement or task (transfer to ballistic finger movement; Experiment 1 and 2), interval range (Experiment 1), target location (Experiment 2), or the VR setting itself (Experiment 3). If temporal perturbations cause global sensorimotor adaptation, we expect to find transfer effects for all tested aspects.

\section{Results}

\section{Experiment 1: Task independency of temporal sensorimotor adaptation}

In a classical sensorimotor adaptation paradigm we asked participants to reproduce intervals by producing two types of movements: In the pointing task participants had to measure and immediately reproduce a $1 \mathrm{~s}$ interval by reaching a target with their right hand (ready-set-go paradigm, see Figure 1A, left column); and, in a similar manner, during the clicking reproduction task participants reproduced intervals ranging between 0.85 and $1.15 \mathrm{~s}$ (in steps of $0.05 \mathrm{~s}$ ) by pressing the trigger button of the controller (see Figure 1A, right column). Test-trials of both tasks were performed before and after an adaptation phase of 40 pointing trials during which the movement was artificially slowed down by a factor of 0.5 , so that participants had to alter their pointing movement in order to sustain performance (e.g., move faster, initiate movement earlier, 
A

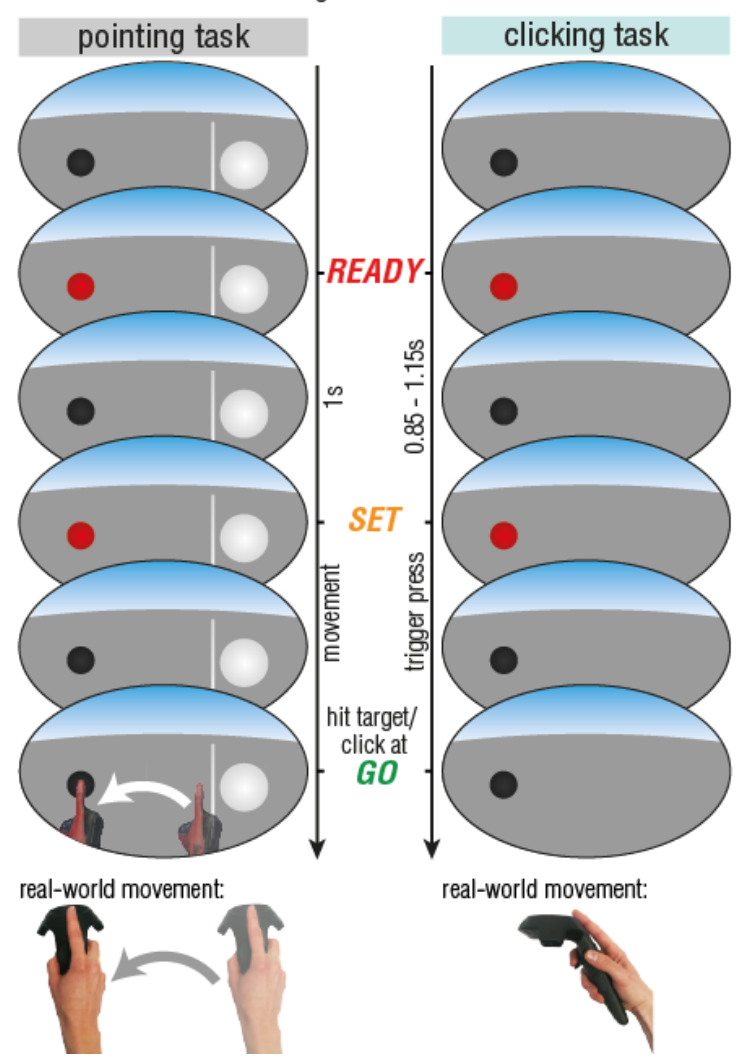

B trial order

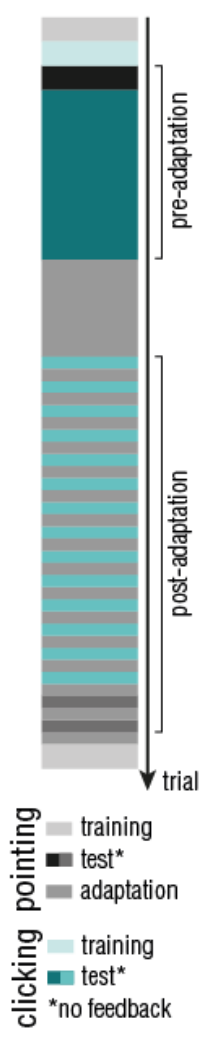

C performance
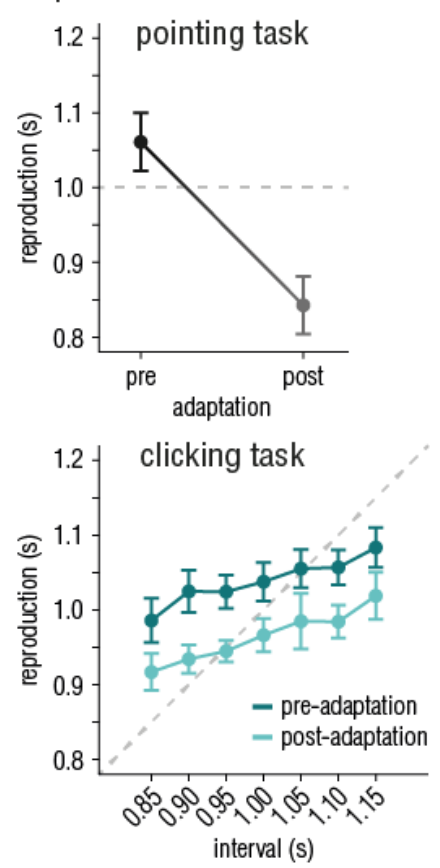

Figure 1. Experiment 1: Task independency of temporal sensorimotor adaptation. Schematic outline of the VR environment (A), the pointing (A, left column) and clicking task (A, right column), trial-structure within one session $(\mathbf{B})$, and behavioral performance $(\mathbf{C})$. A) in a pointing trial, participants had to reproduce the interval marked by the ready- and set-signal by reaching the target (black sphere) in time for the go-signal. Visual feedback was provided by means of a VR-hand, appearing as soon as the movement was initiated. In clicking trials, the interval between the ready- and set-signal had to be reproduced by clicking the trigger button. No additional movement feedback was provided. Participants wore the VR headset at all times. B) Temporal outline and trial order of a single session. C) reproductions in the pointing (top panel) and clicking reproduction task (bottom panel). Data was averaged over all three sessions. Error bars represent $95 \%$ within-subject CIs ${ }^{28,29}$.

change movement trajectory). All participants took part in three sessions of the experiment, separated by at least two hours.

We report Bayes Factors for all analyses as $\mathrm{BF}_{10}$ (i.e., the evidence of the alternative hypothesis over the null hypothesis). We consider $\mathrm{BF}_{10}>3$ as evidence for the alternative hypothesis.

Adaptation phase. The adaptation time course is shown in Figure 2, with separate curves for movement duration (bottom, dark grey), movement onset (middle, medium grey), and interval reproductions (top, light grey). During this adaptation phase movement onset exhibits clear signs of adaptation: Initiation of the pointing movement adapted slowly and gradually over the course of adaptation-trials. Movement duration, in contrast, adapted within a few trials and was retained at this level during the remaining adaptation phase. For both movement onset and duration, a linear model fitted the data best and reflected the gradual decrease in movement duration (slope $=-0.0004$ $\left.\pm 0.0001 \mathrm{SE}, X^{2}(1)=9.53, p<.001, \Delta \mathrm{BIC}=6.26, \mathrm{BF}_{10}=49.86\right)$ and movement onset (slope = $0.002 \pm 0.0003 \mathrm{SE}, X^{2}(1)=36.36, p<.001, \Delta \mathrm{BIC}=30.19, \mathrm{BF}_{10}=50.29$ ) over the course of the 


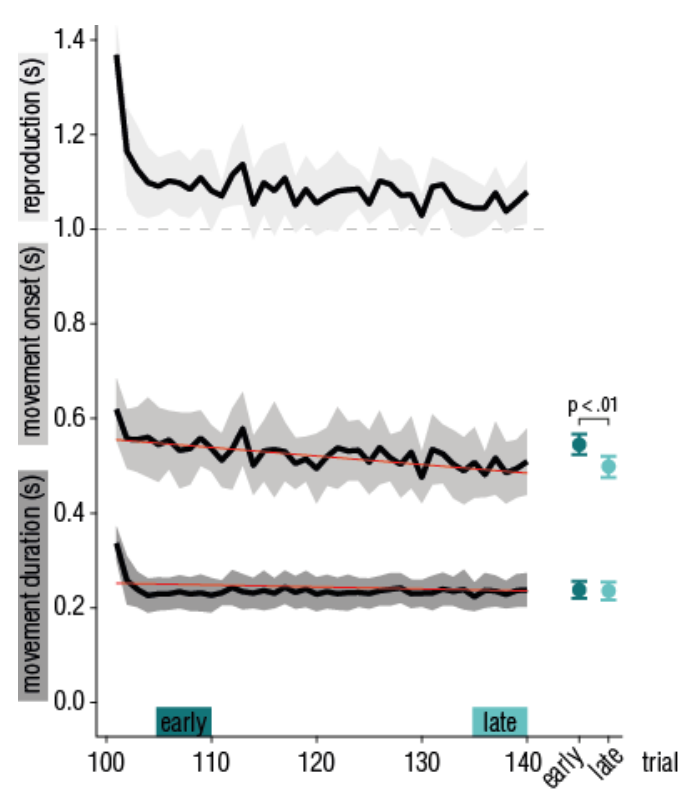

Figure 2. Experiment 1: Adaptation time course. Curves show reproduction (top curve, light grey) and movement start (middle curve, medium grey) were measured relative to the onset of the set-signal. Movement duration (bottom curve, dark grey) was measured from the time the controller passed the start line. Shaded error bars represent 95\% CIs, red lines represent linear fits. Data points on the right represent movement onset (top) and -duration (bottom), averaged over five trials in the early and late phase of adaptation (see colored bars in A), as a measure of adaptation. Error bars represent $95 \%$ within-subject CIs.

adaptation phase. Comparing measures during early (trial 6-10) and late phases of adaptation (trial $36-40)$, we found differences for movement onset $\left(t(11)=3.38, p=.006, d=0.98, \mathrm{BF}_{10}=8.76\right)$, but not for movement duration $\left(t(1)=-0.64, p=.53, d=-0.19, \mathrm{BF}_{10}=0.34\right)$. Adaptation strength was calculated based on pre- and post-movement onset durations ( $M=-0.16,95 \%$ CI [-0.23, 0.10]). The smaller this value, the more sensorimotor adaptation was observed. Because more strongly adapted participants presumably show more adaptation after- or transfer-effects, we incorporated this parameter in the subsequent analyses.

Pointing reproductions. For the analysis of reproduction performance in test trials, depicted in Figure 2C, top panel, we constructed linear mixed models (LMMs) to predict the $1 \mathrm{~s}$ pointing reproductions. The final model to predict reproductions included the factor adaptation strength (coded as 0 for all pre-adaptation trials, and as the above described adaptation strength based on movement onset for all post-adaptation trials; $X^{2}(1)=294.72, p<.001, \Delta \mathrm{BIC}=288.17$, $\left.\mathrm{BF}_{10}>1000\right)$. In other words, $1 \mathrm{~s}$ pointing reproductions in post-adaptation trials were shorter than in pre-adaption trials, and this difference was greater for participants who adapted their movement onset more strongly. Adding the factor session was not warranted $\left(X^{2}(2)=3.16, p=.21, \Delta \mathrm{BIC}=\right.$ $\left.-9.95, \mathrm{BF}_{10}=0.04\right)$, meaning there was no difference in performance between sessions.

To rule out that effects of time-in-experiment (e.g., fatigue) are driving differences in preand post-adaptation performance, we compared RMSEs as a measure of the variable error in preand post-adaptation performance in both tasks. RMSEs were not affected by adaptation $\left(X^{2}(1)=\right.$ $\left.0.78, p=.377, \Delta \mathrm{BIC}=-3.50, \mathrm{BF}_{10}=0.35\right)$. This means that it is unlikely that differences in performance in pre- and post-adaptation trials were driven by time-in-experiment effects. Including session as a predictor was warranted following the likelihood ratio test, however, BIC-values and 
Bayes Factor analysis did not lead to the same conclusion $\left(X^{2}(2)=7.18, p=.028, \Delta \mathrm{BIC}=-1.37\right.$, $\left.\mathrm{BF}_{10}=1.92\right)$. Thus, there is mixed evidence concerning the stability of precision over sessions.

Additional information on pointing trajectories and velocity profiles can be found online at https://osf.io/zbgy9/.

Clicking reproductions. Figure $1 \mathrm{C}$, bottom panel, depicts reproduction performance for the seven different intervals, split on pre- and post-adaption trials. In the LMM analysis, the general trend that longer intervals were reproduced as longer was captured by including the factor interval length $\left(X^{2}(1)=204.48, p<.001, \Delta \mathrm{BIC}=195.98, \mathrm{BF}_{10}>1000\right)$. Regression-towards-the-mean effects were captured by including the duration of the previous trial $\left(\mathrm{N}-1, X^{2}(1)=18.24, p<.001\right.$, $\left.\Delta \mathrm{BIC}=9.74, \mathrm{BF}_{10}=564.51\right)$ and of the trial before the previous $\left(\mathrm{N}-2, X^{2}(1)=6.87, p=.009, \Delta \mathrm{BIC}\right.$ $\left.=-1.62, \mathrm{BF}_{10}=1.9\right)$ in the final model. Note that evidence for the inclusion of the factor $\mathrm{N}-2$ is ambiguous. Apart from this general regression towards the mean, it appears that intervals were systematically underestimated following adaptation-trials of the pointing task. This is reflected in the final model by including the factor adaptation strength $\left(X^{2}(1)=154.59, p<.001, \Delta \mathrm{BIC}=\right.$ $146.10, \mathrm{BF}_{10}>1000$ ), showing that more strongly adapted participants in the pointing task show larger transfer to the clicking task. Together, these results suggest that 1) adaptation effects transferred to another interval reproduction task in which the movement required to produce the go-signal differed substantially from the one that was adapted (clicking instead of pointing); 2) adaptation generalizes to a broader range of intervals; and 3) participants who adapted their motor behavior more strongly in the pointing task also showed larger differences in pre- and postadaptation clicking reproductions. The parallel existence of adaptation and temporal context effects suggests that sensorimotor adaptation and context effect do not interact or cancel each other out, but affect reproductions independently. While temporal context has been shown to already affect the initial temporal estimate ${ }^{14,15}$, its neural origins have been found in motor areas ${ }^{2}$. In neuroimaging studies investigating the locus of sensorimotor adaptation, the cerebellum is, apart from cortical motor areas, thought to play a critical role ${ }^{23}$. Thus, these two effects may originate in different neural substrates (e.g., LIP/SMA vs. cerebellum) or during different processing stages (e.g., perception vs. motor prediction or planning).

We did not find evidence for effects of adaptation $\left(X^{2}(1)=0.76, p=.384, \Delta \mathrm{BIC}=-5.46\right.$, $\left.\mathrm{BF}_{10}=0.14\right)$ or session $\left(X^{2}(2)=5.06, p=.080, \Delta \mathrm{BIC}=-7.39, \mathrm{BF}_{10}=0.24\right)$ on RMSE. Errors adhered to Weber's law ${ }^{30}$ and varied for different intervals $\left(X^{2}(1)=12.78, p<.001, \Delta \mathrm{BIC}=6.56\right.$, $\left.\mathrm{BF}_{10}=49.71\right)$. As for the analysis of RMSEs in the pointing task, these results speak against an interpretation of pre-post-adaptation performance differences being driven by, for example, increased fatigue over the course of the experiment.

Transfer. To quantify the amount of adaptation transfer from the pointing to the clicking reproduction task, we calculated transfer as the difference between pointing reproductions in testtrials before and after adaptation with the change in clicking reproductions induced by the adaptation procedure. In this experiment, $41.00 \%$ (95\% CI [18.59, 63.41]) of the adaptation effect in the pointing task transferred to the clicking task, which, tested with a one-sample $t$-test, differed significantly from zero $\left(t(11)=4.03, p=.002, d=1.16, \mathrm{BF}_{10}=22.41\right)$. 


\section{Experiment 2: Location independency of temporal sensorimotor adaptation}

Results of Experiment 1 revealed that sensorimotor adaptation affected participants' timing abilities and transferred to another type of movement. As already touched upon in the introduction, sensorimotor adaptation effects are often reported to exhibit spatial selectivity, that is, adaptation effects are observed only in the region in which the adapter was shown or the adaptation movement was executed. In Experiment 1, the coordinates of the target (the black sphere) are the same in both tasks and in all kinds of trials. To test in how far adaptation effects in our VR setup are location specific, we changed the target position in test-trials of the pointing and/or the clicking task in Experiment 2 (see Figure 3A), meaning that in some cases the target in test trials appeared mirrored compared to adaptation trials. The four different task-target-location combinations were tested in four separate sessions (Figure 3B). Adaptation phase analyses, pointing trajectories and velocity profiles can be found at https://osf.io/zbgy9/.

Pointing reproductions. Analogue to the model analysis performed for pointing reproductions in Experiment 1, including the factor adaptation strength $\left(M_{\text {same location }}=-0.13,95 \%\right.$ CI $[-0.26,0] ; M_{\text {different location }}=-0.01,95 \%$ CI $\left.[-0.14,0.1]\right)$ to predict interval reproductions in the pointing task improved the model fit $\left(X^{2}(1)=17.32, p<.001, \Delta \mathrm{BIC}=11.01, \mathrm{BF}_{10}=466.87\right)$. To test whether target location in test trials affected reproductions additionally, we included the factor location (coded as "same" or "different" compared to adaptation trials). Location did, however, not improve the model fit $\left(X^{2}(1)=1.27, p=.26, \Delta \mathrm{BIC}=-5.05, \mathrm{BF}_{10}=0.18\right)$. This shows that, regardless of whether the target in test-trials appeared at the same location as in adaptation-trials, reproductions were influenced by sensorimotor adaptation in that reproductions were systematically shorter in post-adaptation compared to pre-adaptation test-trials (Figure 3C, top panel).

There was no evidence for effects of adaptation $\left(X^{2}(1)<.01, p=.995, \Delta \mathrm{BIC}=-3.33\right.$, $\left.\mathrm{BF}_{10}=0.34\right)$ or location $\left(X^{2}(1)=0.10, p=.755, \Delta \mathrm{BIC}=-3.23, \mathrm{BF}_{10}=0.36\right)$ on RMSEs, ruling out time-in-experiment effects or location dependent training effects.

Clicking reproductions. Figure 3C, bottom panel, depicts reproduction performance, split on pre- and post-adaption trials and on the location of the target. The final model included the predictor adaptation strength $\left(\left(M_{\text {same location }}=-0.08,95 \%\right.\right.$ CI $[-0.23,0.06] ; M_{\text {different location }}=-0.06$, $\left.95 \% \mathrm{CI}[-0.21,0.09]), X^{2}(1)=17.32, p<.001, \Delta \mathrm{BIC}=11.01, \mathrm{BF}_{10}=488.11\right)$, while the location of the target

did not improve the model fit $\left(X^{2}(1)=1.25, p=.26, \Delta \mathrm{BIC}=-5.07, \mathrm{BF}_{10}=0.18\right)$. Thus, intervals in the clicking task were systematically under-reproduced after adapting to the altered temporal properties in the pointing task. As for the pointing task, effects of adaptation on clicking reproductions are independent of location.

We did not find evidence for effects of adaptation $\left(X^{2}(1)=1.83, p=.176, \Delta \mathrm{BIC}=-1.50\right.$, $\left.\mathrm{BF}_{10}=0.69\right)$ or location $\left(X^{2}(1)=1.76, p=.185, \Delta \mathrm{BIC}=-1.57, \mathrm{BF}_{10}=0.66\right)$ on RMSEs.

Transfer. Because we did not find evidence for an effect of location on pointing reproductions, nor on clicking reproductions, we averaged over all location conditions for the calculation of transfer from adaptation in the pointing to the clicking task. The amount of adaptation transfer was $59.62 \%\left(95 \%\right.$ CI $\left.[23.99,95.49], t(6)=4.09, p=.006, d=1.55, \mathrm{BF}_{10}=9.49\right)$. Adapting 
A

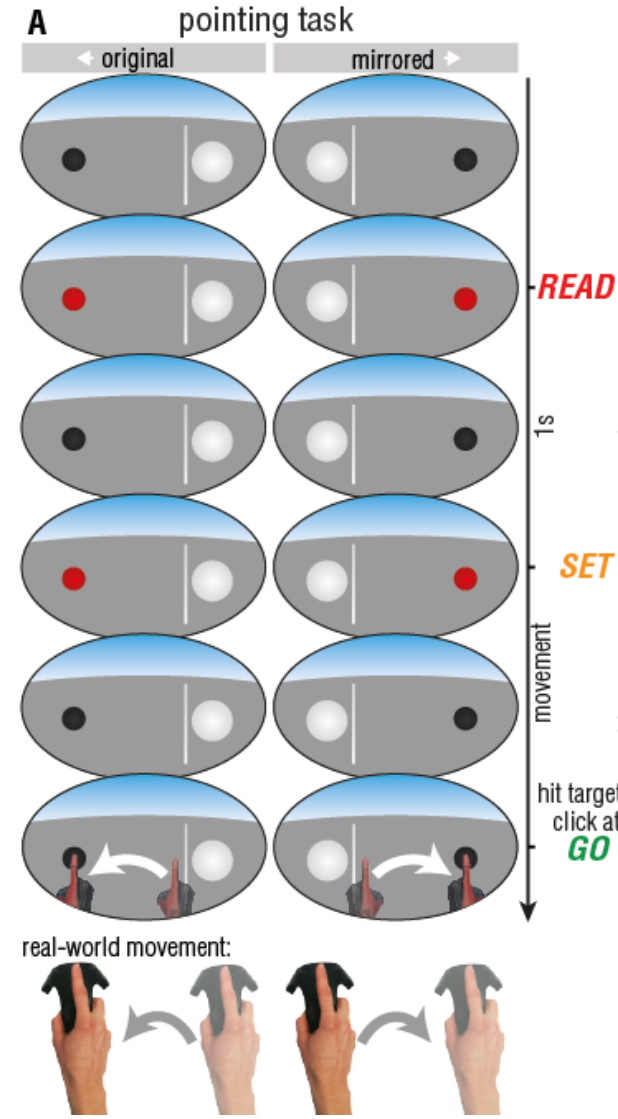

clicking task
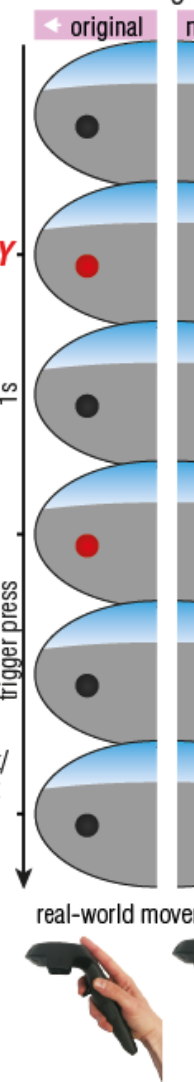

B
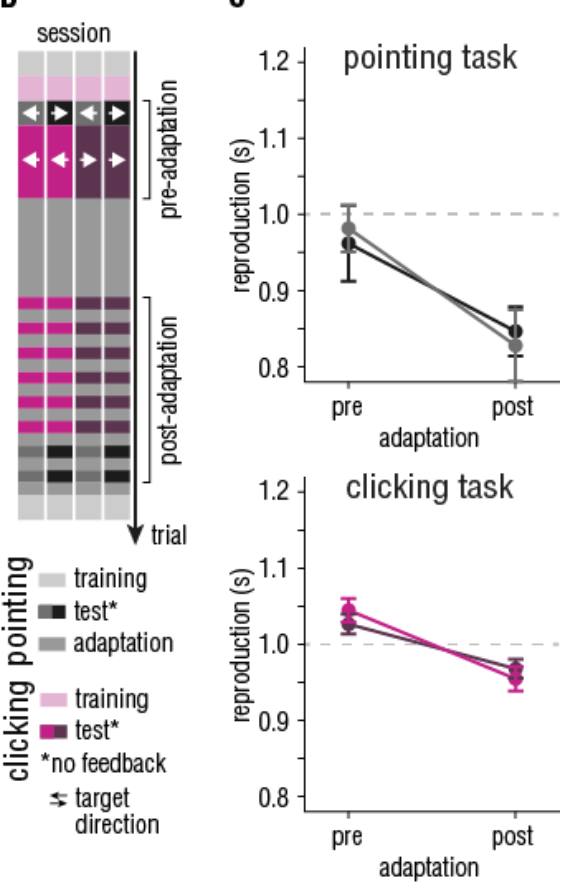

- target $_{\text {test }}=$ target $_{\text {adaptation }}$ - target $_{\text {test }} \neq$ target $_{\text {adaptation }}$

Figure 3. Experiment 2: Location independency of temporal sensorimotor adaptation. Schematic outline of the VR environment (A), the pointing (A, left column, mirrored and original) and clicking task (A, right column), trialstructure within one session $(\mathbf{B})$, and behavioral performance $(\mathbf{C})$. $\mathbf{A})$ in a pointing trial, participants had to reproduce the interval marked by the ready- and set-signal by reaching the target (black sphere) in time for the go-signal. Visual feedback was provided by means of a VR-hand, appearing as soon as the movement was initiated. In clicking trials, the interval between the ready- and set-signal had to be reproduced by clicking the trigger button. No additional movement feedback was provided. The location and pointing direction in test trials varied (original and mirrored). Participants wore the VR headset at all times. B) Temporal outline and trial order of the four different sessions. C) Reproductions in the pointing (top panel) and clicking reproduction task (bottom panel). Data was pooled together depending on whether the target changed location in the pointing task for pointing reproductions, or whether the target changed location in the clicking task for clicking reproductions. Error bars represent 95\% within-subject CIs

to altered temporal aspects of timed actions seems to affect all motor planning regarding direction or type of movement.

\section{Experiment 3: Environment independency of temporal sensorimotor adaptation}

In the previous experiments we found that sensorimotor adaptation to temporal perturbations generalizes to all motor actions aimed to reproduce a given interval. These results, however, could be caused by a general correction of movements to overcome the temporal lag associated to the VR environment, and not by adaptation of the motor system. To rule out that the above described findings apply to VR only, we tested whether adaptation effects also transfer to a pointing task outside of VR. In the real-world task, participants had to point to a green LED, attached to an Arduino microcontroller and controlled by Unity, which was positioned at the same 
location as the target in the VR environment (see schematic outline in Figure 4A). Adaptation phase analyses, pointing trajectories and velocity profiles can be found at https://osf.io/zbgy9/.

Pointing reproductions. While including the factor adaptation strength to predict reproductions in the pointing task did not improved the model fit ( $M_{V R}=-0.01$ (95\% CI [-0.21, $\left.0.18]), M_{\text {real-world }}=-0.13(95 \% \mathrm{CI}[-0.32,0.07]), X^{2}(1)=3.63, p=.06, \Delta \mathrm{BIC}=-1.64, \mathrm{BF}_{10}=0.96\right)$, including a binary factor adaptation (coded as "pre" and "post") did significantly improve model fits $\left(X^{2}(1)=58.70, p<.001, \Delta \mathrm{BIC}=53.43, \mathrm{BF}_{10}>1000\right)$. Including a factor encoding environment (coded as "VR" or "real-world") was not warranted $\left(X^{2}(1)=0.02, p=.90, \Delta \mathrm{BIC}=-5.26, \mathrm{BF}_{10}=\right.$ 0.14). This shows that, regardless of whether the pointing task was performed within the VR

A

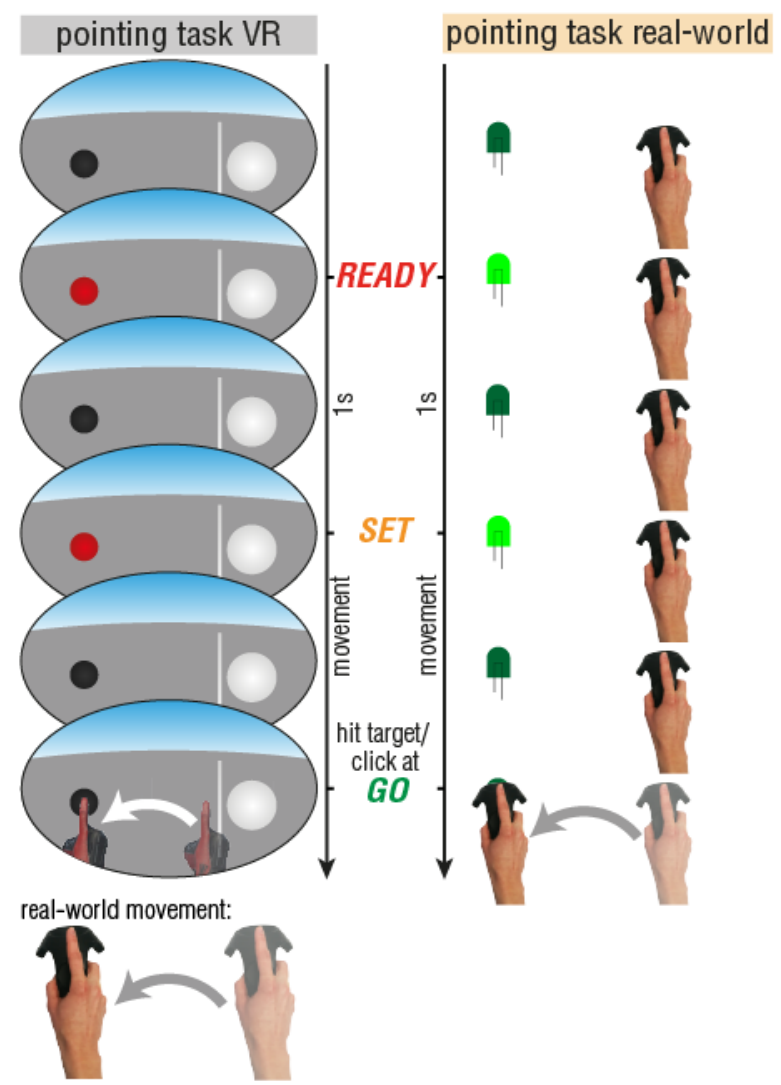

B trial order

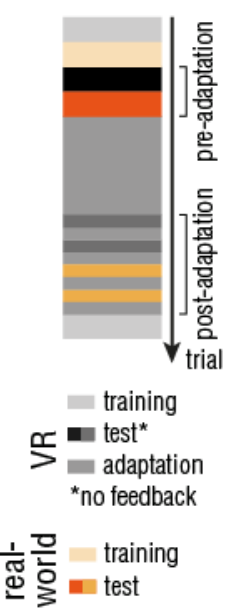

C performance
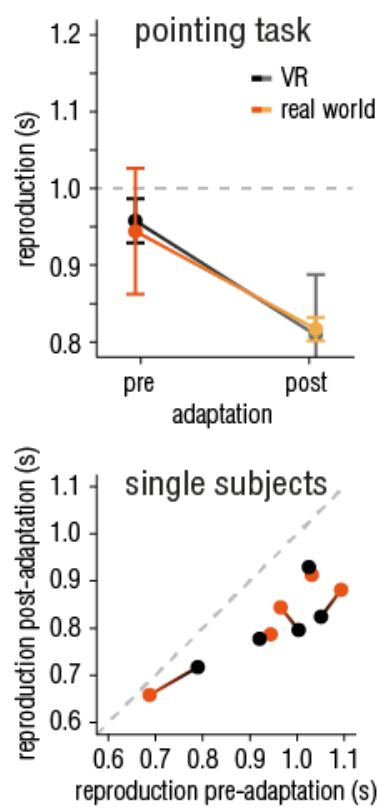

Figure 4. Experiment 3: Environment independency of temporal sensorimotor adaptation. Schematic outline of the VR environment (A, left column), the pointing task in VR (A, left column) and pointing task in the real-world (A, right column), trial-structure (B), and behavioral performance (C). A) Participants had to reproduce the interval marked by the ready- and set-signal by reaching the target (black sphere in VR, green LED in real-world) in time for the gosignal. In VR, visual feedback was provided by means of a VR-hand, appearing as soon as the movement was initiated. In real-world, participants could see their hand. Participants wore the VR headset dependent on task requirements. B) Temporal outline and trial order. C) Averaged reproductions in the pointing task separated for VR and real-world task (top panel). Influence of adaptation in single subject data separated for VR and real-world task (connected dots represent one subject), plotted as pre-adaptation reproductions against post-adaptation reproductions. Dots below the dashed line reflect effects of adaptation (under-reproduction in post-adaptation trials compared to pre-adaptation trials), and the distance to the dashed line reflects the strength of adaptation (larger distance $\sim$ larger adaptation effect). Error bars represent $95 \%$ within-subject CIs. 
environment or not, reproductions were influenced by sensorimotor adaptation, irrespective of how strongly participants were adapted (Figure 4C).

There was no evidence for effects of adaptation $\left(X^{2}(1)=0.95, p=.33, \Delta \mathrm{BIC}=-2.05\right.$, $\left.\mathrm{BF}_{10}=0.55\right)$ or $\mathrm{VR}\left(X^{2}(1)=0.21, p=.65, \Delta \mathrm{BIC}=-2.79, \mathrm{BF}_{10}=0.43\right)$ on RMSEs, ruling out timein-experiment effects or environment dependent effects.

Transfer from VR to the real world. The amount of adaptation transfer from VR to nonVR was $85.16 \%$ (95\% CI [41.7, 128.62], $\left.t(4)=5.44, p=.006, d=2.43, \mathrm{BF}_{10}=10.81\right)$. Adaptation was not contextually cued by being in the VR environment or by wearing VR goggles. Instead, the crucial component driving the transfer of adaptation effects from VR to the real world may be the movement goal - reaching the target at the go-signal - which was the same within VR and the real world. The finding of substantial transfer further confirms the presence of sensorimotor adaptation, because, unlike in VR test-trials, participants can see their hand in the analogue task and thus have immediate visual feedback on their movement time course.

\section{Discussion}

The interconnectedness of the motor system and the processing of time has been well established over the last couple of years ${ }^{6-11}$. However, temporal estimates and movements are often experimentally kept apart. In the current study, we explored the temporality of movements as such (i.e., temporal estimates were directly contingent on movement dynamics), and how altering the temporality of a specific movement alters the temporality of other movements. In a set of three experiments, we tested whether adapting to temporal perturbations for a specific movement embedded in a specific context transfers to movements other than the adapted movement and to contexts other than the adapted context. Participants had to adjust their pointing movement required to reproduce an interval to cope with slowed down hand movements in a VR environment. Over the course of this adaptation phase participants incorporated performance feedback and gradually varied movement onset (i.e., started the movement earlier) and speeded up their movements (i.e., decreased movement duration). This adaptation affected subsequent pointing reproductions, causing systematic under-reproductions once the temporal perturbation was removed.

We found that adaptation transfers from continuous arm reaching movements to ballistic finger movements. Continuous and ballistic movements differ drastically in the requirements of their control. Continuous movements are monitored online to regulate their speed and duration in order to steer the hand to the desired goal location. For ballistic movements, like clicking, only the onset can be variably controlled whereas the movement itself, once initiated, underlies an automatic routine. Since timing adaptation affected the planning of movements with such different dynamics it seems as if the motor system as a whole (or for the right arm) adapted to the temporal perturbation. We also found that adaptation effects transferred to a pointing movement in the opposite direction, ruling out that adaptation is specific to location. The observation that manipulated visual feedback during the adaptation phase causes adaptation effects independent of task and target location adds to the evidence that temporal measurements are grounded in, or at least intimately linked to, action and the motor system.

An often highly debated aspect of adaptation paradigms is whether changes in behavior occur because of cognitive strategies or implicit learning - while the simultaneous existence of 
both is generally agreed upon ${ }^{20,23,31-37}$. Attempts to disentangle the two rely on preventing the use of explicit strategies (e.g., by debriefing participants about the perturbation before the experiment and instructing them to ignore the manipulated feedback ${ }^{32,35}$ ), or by inquiring about explicit strategies (e.g., asking about their aim-point before the pointing movement in spatial adaptation $^{31,37}$ ). In all these studies, regardless of how obvious or abrupt the artificial perturbation was introduced, signs of adaptation in the absence of explicit cognitive strategies were observed and were attributed to implicit adaptation. The current study did not attempt to distinguish between implicit and explicit adaptation processes. Thus, any observed effects of adaptation could stem from either explicit or implicit processes, or from a mixture of both. In fact, given that participants receive precise feedback on their performance during the adaptation phase in all classical adaptation paradigms, it would be surprising if this feedback was not consciously and explicitly used to alter movements in order to sustain performance. However, there are a few reasons why we think that implicit adaptation occurred alongside of cognitive strategies. In visuomotor and prism adaptation commonly found signatures of implicit adaptation are a gradual adaptation timecourse and the existence of aftereffects ${ }^{33,38}$. We found both of these features in our data: After a fast, initial movement adaptation, participants continued to gradually adapt to the temporal distortion (see Figure 2). Additionally, we found clear signs of adaptation aftereffects in all three experiments. Aftereffects even persisted in the real-world pointing task during which participants had online feedback about the trajectory of their hand, inviting them to consciously recalibrate their movement. While participants most likely used conscious strategies during the first trials of the adaptation phase, the above summarized observations are evidence that implicit adaptation must have occurred alongside and over the course of the adaptation phase.

Typically, transfer of adaptation is highly specific to the particular movement (i.e., movement type and direction, context, effector, or task) that is trained in the adaptation trials. This is true for adaptation to spatial ${ }^{23}$ and spatiotemporal distortions ${ }^{19}$ (e.g., intercepting moving targets with delayed movement feedback), as well as for motor-aftereffects on temporal judgements (e.g., the speed of finger tapping influences perceived duration in a subsequent visual interval discrimination task ${ }^{6,39-42}$ ). In the current study, temporal sensorimotor adaptation effects were not spatially selective, and adaptation effects persisted regardless of the type of movement (continuous pointing or ballistic clicking). Adaptation effects even persisted outside of the VR environment, ruling out that participants altered their behavior because of the motion-to-photon latency of the VR system and that adaptation effects were object-based (i.e., tied to the target in the VR environment). In other words, the locus of adaptation effects was neither (fully) extrinsic, nor (fully) object-centered. Instead, observed effects of adaptation were intrinsic and affected temporal predictions and temporal planning of all goal directed motor actions (i.e., motor actions performed to reproduce an interval) tested in the study. Future studies could further test the transferability of temporal sensorimotor adaptation. For example, the transfer from one to the other limb, because different effectors have been associated with separate motor-clocks in other tasks ${ }^{43}$, or the transfer from a visual to an auditory event, because auditory and visual time are thought to be processed modality specific ${ }^{44}$. In how far sensory predictions of durations are affected by sensorimotor adaptation remains to be tested, too. 
Because environments and tasks are rarely as simplistic as in the current study, adapting motor actions to temporal perturbations globally ensures that complex and time-critical behavior can be executed successfully. Encoding temporal estimates within the motor system and recalibrating all motor actions aids to produce optimally timed behavior and increase ecological fitness. As an illustrative example, imagine the following fictious event: The $100 \mathrm{~m}$ sprint runner from the initial example was injected with a local, immediately effective doping substance in the left hamstring, which is now reacting and moving much faster. A sprint start involves a complex sequence of movements that need to be attuned to each other. In order to keep the movement sequence of the start routine intact after the injection all motor plans need to adapt to the altered temporal behavior of the left hamstring, so that the runner still leaves the starting block on the gosignal. If each muscle would need to recalibrate separately, hopes for the gold medal will probably fade after a poor start of the sprinter. Instead, we showed that sensorimotor adaptation to temporal perturbations affects temporal motor predictions and temporal motor planning globally, allowing for smooth and well-timed motor actions. Unlike in spatial adaption, where only specific movements to a specific target coordinate is subject to adaptation-effects, adaptation to temporal perturbations transfers to different tasks involving different movements, different target locations, and different environmental contexts. Indeed, coordinating behavior in time should not be specific to movement type or movement goal, because the timing of movements can be detached from spatial features, like different trajectories for different movement types or different target locations. For an agent interacting with its environment, time may thus be an ubiquitous property that is inherently rooted within the motor system.

\section{Materials \& Methods}

\section{Experiment 1: Task independency of temporal sensorimotor adaptation}

Apparatus. The experiments were conducted on a Windows 10 based desktop computer (Alienware Aurora R8, Intel(R) Core ${ }^{\mathrm{TM}}$ i7-8700 CPU @ $3.20 \mathrm{GHz}, 16$ GB RAM, NVIDIA GeForce GTX 1080TI graphics card) connected to an HTC Vive Pro Eye Head Mounted Display (HMD) (HTC Corporation, Taoyuan, Taiwan). The HMD presents stimuli on two low-persistence organic light-emitting diode (OLED) displays with a resolution of 1,440 x 1,600 pixels per eye and a refresh rate of $90 \mathrm{~Hz}$. Additionally, participants used a Vive motion-controller for their right hand. The virtual environment was rendered using SteamVR 1.10 and a custom-made program created in Unity game engine, version 2019.1.13f1 (Unity Technologies, San Francisco, U.S.). Head and hand movements were tracked via the HMD and controller using the SteamVR 1.0 tracking system. According to previous research, this system provides a robust tracking of head and hand motion with a $360^{\circ}$ coverage, provided tracking loss is prevented ${ }^{45}$. Tests of Verdelet et al. ${ }^{46}$ demonstrated a submillimeter precision $(0.237 \mathrm{~mm})$ and an accuracy of $8.7 \mathrm{~mm}$ for static and $8.5 \mathrm{~mm}$ for dynamic objects. While the system can update the user's pose (position and orientation) at a higher rate (up to $1000 \mathrm{~Hz}$ for the HMD and $250 \mathrm{~Hz}$ the controllers), in this study the sampling rate for both HMD and controller was limited by the HMD's refresh rate of $90 \mathrm{~Hz}$.). Because participants always responded to stimuli in front of them, we did not need the full coverage around participants in the present study. Hence, in order to minimize the chance of occlusions of the HMD or controller and thereby avoiding tracking loss, our setup had both base stations facing the participant. Throughout 
the experiment, participants held the controller with an outstretched index finger placed on top of the controller with the fingertip matching the tracking origin of the controller as close as possible (see Figure 1A). Participants remained seated during the entire experiment. The apparatus is the same for all reported experiments.

Participants. 12 participants (8 female, 4 authors, age range 19-42 years, all right-handed) were tested. Sample size in all experiments was based on previous similar research (e.g., 5-8 participants per experiment in Anobile et al. ${ }^{6}$ ). All participants had normal or corrected-to-normal vision. Participants gave informed consent prior to participation. The experiments were carried out along the principles laid down in the Declaration of Helsinki. All experiments were approved by the local ethics committee of the psychological department of the Heinrich-Heine-University Düsseldorf.

Pointing reproduction task. In the pointing task participants had to measure and immediately reproduce a $1 \mathrm{~s}$ interval by reaching a target with the hand controller (ready-set-go paradigm, see Figure 1A, left column). At the beginning of the trial participants had to place the controller behind the start line in a sphere (diameter $=10 \mathrm{~cm}$ ), which was located slightly to their right bottom at $\mathrm{x}=20 \mathrm{~cm}, \mathrm{y}=-40 \mathrm{~cm}$, and $\mathrm{z}=30 \mathrm{~cm}$, with respect to their head position $(\mathrm{x}=0, \mathrm{y}$ $=0, \mathrm{z}=0 \mathrm{~cm}$ ). To their left they saw a small black sphere, the target (diameter $=3 \mathrm{~cm}, \mathrm{x}=-15, \mathrm{y}$ $=-40, \mathrm{z}=30 \mathrm{~cm}$, distance between start line and target was set to $30 \mathrm{~cm})$. The target changed its color to red for $0.1 \mathrm{~s}$, first to mark the start of the interval presentation (ready) and again after $1 \mathrm{~s}$ to mark the end of the interval presentation and the start of the reproduction (set). Participants had to virtually touch the sphere to end their reproduction ( $\mathrm{go}$ ). As soon as participants crossed the start line with the controller, they saw a VR-hand following the movement of their physical hand. We will refer to the time between crossing the start line and reaching the target as movement duration. Participants received immediate feedback on their performance: The deviation of participants' reproduction from the target interval (a negative number corresponded to under-reproductions) was displayed above the target, additionally color-coded in red (deviation $>0.3$ s), yellow $(0.1>$ deviation $<0.3 \mathrm{~s}$ ), or green (deviation $<0.1 \mathrm{~s}$ ). The next trial started once participants moved their hand back to the start position and pressed the trigger-button of the motion-controller with their middle finger. We used three different kinds of trials: Training-trials, adaptation-trials, and testtrials (see Figure 1B for a temporal outline of the experiment). Training-trials were as described above and used to accustom participants to the VR environment and for de-adaptation at the end of the experiment. In adaptation-trials participants saw the VR-hand move at half the speed of their actual movement, that is, participants received delayed visual feedback by means of the VR hand. The reproduction of the $1 \mathrm{~s}$ interval depended on the VR-hand reaching the sphere with the index finger, so that for an accurate reproduction, participants had to adapt their movement by speeding up the pointing action through implicit motor learning. An alternative strategy is to start the hand movement earlier without changing movement speed, or to use a combination of both faster movement and earlier movement start. Changes in movement speed are thought to reflect implicit adaptation, while changes in movement onset are thought to be more cognitively controlled ${ }^{23}$. Feedback was given on every trial. In test-trials participants did not see the VR-hand movement and received no feedback. 
Clicking reproduction task. For the clicking task, we used the same ready-set-go paradigm as in the adaptation task. Participants ended their reproductions (i.e., indicate the go-signal) by pressing the trigger button of the controller (see Figure 1A, right column). Tested intervals ranged between $0.85 \mathrm{~s}$ and $1.15 \mathrm{~s}$ in steps of $0.05 \mathrm{~s}$. In training-trials participants received feedback as described above. In test-trials participants received no feedback on their performance.

Procedure. Figure 1B depicts the temporal outline of the experiment. Each participant completed the experiment three times in three sessions, separated by at least 4 hours. In each session participants first got accustomed to the VR environment and the tasks by completing 10 training-trials of both tasks. In the pre-adaptation phase, each participant completed 10 test-trials of the pointing task and 70 test-trials of the clicking task (i.e., each interval was presented 10 times). This first test phase was followed by 40 adaptation-trials of the pointing task. The 70 postadaptation clicking reproduction task trials were interleaved with adaptation trials of the pointing task (five trials each). This was followed by 10 test-trials of the pointing task, again interleaved with adaptation-trials. At the end of the experiment, participants completed 10 more training-trials of the pointing task to de-adapt.

Statistical analysis. As an individual measure of the magnitude of motor adaptation we calculated the proportional change in the averaged movement onset in pre- versus post-adaptation

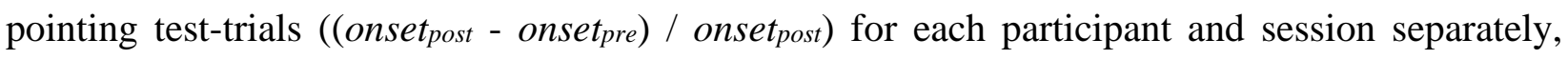
reflecting the amount of implicit motor learning in each participant and session. Movement onset was identified as the most likely candidate to quantify implicit adaptation. This was tested by fitting linear and exponential models and comparing their performance in predicting development of movement onset and movement duration over the course of the adaptation phase. Additionally, we compared the average movement duration and movement onset of five trials in the beginning (omitting the very first five trials of the adaptation phase, so that fast, strategic recalibration does not obscure this measure) and at the end of the adaptation phase (Figure 3, right) by means of twosided $t$-tests. A difference between early and late adaptation phase would hint at gradual changes in behavior over the course of trials.

Participants' behavior in the pointing reproduction task was analyzed by means of LinearMixed-Models (LMMs) regression using the $1 m e 4^{47}$ and 1 merTest ${ }^{48}$ packages in $\mathrm{R}$ version 4.0.3 ${ }^{49}$. Models were constructed to predict the reproduced $1 \mathrm{~s}$ interval with predictors including adaptation (coded as 0 for all pre-adaptation trials and as the difference in movement onset in pre- and postadaption trials in the pointing task, dependent on participant and session, see above) and session (1-3, as factor). In all models, intercepts varied by participant. All models with different combinations of these predictors, with or without interactions between main effects, were compared by means of likelihood ratio tests, BIC values (we consider a reduction of 10 as evidence to include a given factor) and Bayes Factors ( $\mathrm{BF}_{10}$; throughout the manuscript we report the $\mathrm{BF}$ as the evidence of the alternative hypothesis over the null hypothesis; we consider $\mathrm{BF}_{10}>3$ as evidence for the alternative hypothesis) calculated using the BayesFactor package ${ }^{50}$. We report the resulting best model and statistical evidence for or against effects.

Models that were constructed to predict participants' behavior in the clicking reproduction task incorporated the following predictors: Interval length (i.e., the to-be-reproduced interval, zerocentered), previously presented durations (i.e., interval duration of trial $\mathrm{N}-1, \mathrm{~N}-2$, etc.), adaptation 
(see above), and session (see above). In all models, intercepts varied by participant. We proceeded with model selection as described above.

To rule out that effects of time-in-experiment (e.g., fatigue) are driving differences in preand post-adaptation performance, we compared root mean squared errors (RMSEs), calculated from an estimated linear slope and scaled by mean reproduction for each participant ${ }^{51}$, as a measure of the variable error in pre- and post-adaptation performance in both tasks. This test was based on the rationale that with increasing fatigue, reproductions in post-adaptation trials should become more variable than in pre-adaptation trials. For the analysis, we constructed models with predictors including: Interval length (for the clicking task only, coded as described above), session, and adaptation (coded as described above). In all models, intercepts varied by participant.

Finally, to quantify the amount of transfer from the adapted pointing to the clicking reproduction task, we compared the difference between pointing reproductions in test-trials before and after adaptation with the change in clicking reproductions induced by the adaptation procedure:

$$
\text { transfer }=\frac{\left(\text { pointingreproduction }_{\text {post }}-\text { pointingreproduction }_{\text {pre }}\right)}{\left(\text { clickingreproduction }_{\text {post }}-\text { clickingreproduction }_{\text {pre }}\right)} \times 100
$$

Trials in which the reproduced duration was shorter or longer than 3 median absolute deviations were excluded from the analysis ${ }^{52}$. This led to the exclusion of $2.2 \%$ of trials in the pointing task and $2.6 \%$ of trials in the clicking task.

\section{Experiment 2: Location independency of temporal sensorimotor adaptation}

Participants. 7 participants (4 female, 3 authors, age range 23-42 years, all right-handed) who already participated in Experiment 1 and 2 were re-tested in Experiment 2.

Pointing reproduction task. The pointing task was essentially the same as in Experiment 1 (Figure 4A, left column), with the only differences that the visual scene for test trials was mirrored in two out of four sessions (i.e., in mirrored sessions participants had to point from left to right, Figure 4B).

Clicking reproduction task. The clicking task was essentially the same as in Experiment 1 (Figure 4A, right column), with the only differences that we used only the 1 s interval; and that the visual scene for test trials was mirrored in two out of four sessions (Figure 3B).

Procedure. Each participant completed four sessions, separated by at least two hours. Sessions differed in the way mirrored conditions were combined: Test trials were mirrored 1) for both tasks, 2) for the adaptation task only, 3) for the interval reproduction task only, or 4) for none of the tasks. Trial structure was identical to Experiment 1, with the only exception that only 30 preand post-adaptation clicking test-trials were performed. The temporal outline of the experiment is depicted in Figure 3C.

Statistical analyses. Analysis for the location dependent motor adaptation experiment was identical to Experiment 1, with the only exception that 'interval' was not included as a predictor in any model, and we added the predictor location (coding for whether the target in test-trials appeared at the same or different location than in adaptation-trials). $0.9 \%$ of trials in the pointing task and $6.7 \%$ of trials in the clicking task were excluded from the analysis (see Experiment 1 for exclusion criteria). 


\section{Experiment 3: Environment independency of temporal sensorimotor adaptation}

Participants. 5 participants (3 female, 2 authors, age range 23-36 years, all right-handed) who already participated in Experiment 1, 2 and 3 were re-tested in Experiment 3.

Pointing reproduction task. The pointing task was the same as in Experiment 1, with the only differences that an additional set of 10 test-trials were performed outside of the VR environment (see Figure 4A and B). For the real-world pointing task, a green LED, attached to an Arduino microcontroller controlled by Unity, was positioned in the physical location directly below the virtual target. To ensure that participants were able to find the start position in the non-VR condition, a blue LED was attached to the Arduino and lit up when the hand was overlapping with the virtual start position.

Procedure. This experiment comprised one session only. Participants first got accustomed to the VR environment and the task by completing 10 training-trials. In the pre-adaptation phase, each participant completed 10 test-trials of the pointing task within and outside of the VR environment. The pre-adaptation test phase was followed by 40 adaptation-trials. In the postadaptation phase participants again performed 10 test trials of the pointing task within and outside of the VR environment, interleaved with adaptation-trials. At the end of the experiment, participants underwent the de-adaptation procedure as in the other experiments.

Statistical analyses. Analysis for the VR-dependent motor adaptation experiment was identical to Experiment 2, with the exception that instead of the predictor 'location' we included the binary predictor 'VR'. The transfer from adaptation within VR to adaptation outside VR was calculated following formula (1). No trials had to be excluded from the analysis (see Experiment 1 for exclusion criteria).

\section{Data availability}

All data and analysis scripts are available online at https://osf.io/zbgy9/.

\section{References}

1. Remington, E. D., Parks, T. V. \& Jazayeri, M. Late Bayesian inference in mental transformations. Nat Commun 9, 4419 (2018).

2. Jazayeri, M. \& Shadlen, M. N. A neural mechanism for sensing and reproducing a time interval. Current Biology 25, 2599-2609 (2015).

3. Ivry, R. B. The representation of temporal information in perception and motor control. Current Opinion in Neurobiology 6, 851-857 (1996).

4. Safaie, M. et al. Turning the body into a clock: Accurate timing is facilitated by simple stereotyped interactions with the environment. Proc Natl Acad Sci USA 117, 13084-13093 (2020).

5. Buzsáki, G. The brain from inside out. (Oxford University Press, 2019).

6. Anobile, G., Domenici, N., Togoli, I., Burr, D. \& Arrighi, R. Distortions of visual time induced by motor adaptation. Journal of Experimental Psychology: General 149, 1333-1343 (2020).

7. Tomassini, A., Vercillo, T., Torricelli, F. \& Morrone, M. C. Rhythmic motor behaviour influences perception of visual time. Proc. R. Soc. B. 285, 20181597 (2018). 
8. Yokosaka, T., Kuroki, S., Nishida, S. \& Watanabe, J. Apparent time interval of visual stimuli is compressed during fast hand movement. PLoS ONE 10, e0124901 (2015).

9. Yon, D., Edey, R., Ivry, R. B. \& Press, C. Time on your hands: Perceived duration of sensory events is biased toward concurrent actions. Journal of Experimental Psychology: General 146, 182-193 (2017).

10. Ueda, S. \& Shimoda, S. Subjective time compression induced by continuous action. Sci Rep 11, 13488 (2021).

11. Hagura, N., Kanai, R., Orgs, G. \& Haggard, P. Ready steady slow: Action preparation slows the subjective passage of time. Proc. R. Soc. B. 279, 4399-4406 (2012).

12. Van Rijn, H. Accounting for memory mechanisms in interval timing: A review. Current Opinion in Behavioral Sciences 8, 245-249 (2016).

13. Sohn, H., Narain, D., Meirhaeghe, N. \& Jazayeri, M. Bayesian computation through cortical latent dynamics. Neuron 103, 934-947.e5 (2019).

14. Damsma, A., Schlichting, N. \& Van Rijn, H. Temporal context actively shapes EEG signatures of time perception. bioRxiv preprint (in press) doi:10.1101/2020.03.17.995704.

15. Zimmermann, E. \& Cicchini, G. M. Temporal context affects interval timing at the perceptual level. Sci Rep 10, 8767 (2020).

16. De Kock, R., Zhou, W., Joiner, W. M. \& Wiener, M. Slowing the body slows down time perception. eLife 10, e63607 (2021).

17. Botzer, L. \& Karniel, A. Feedback and feedforward adaptation to visuomotor delay during reaching and slicing movements. Eur J Neurosci 38, 2108-2123 (2013).

18. Cunningham, D. W., Chatziastros, A., von der Heyde, M. \& Bülthoff, H. H. Driving in the future: Temporal visuomotor adaptation and generalization. Journal of Vision 1, 3 (2001).

19. de la Malla, C., Lopez-Moliner, J. \& Brenner, E. Dealing with delays does not transfer across sensorimotor tasks. Journal of Vision 14, 8-8 (2014).

20. López-Moliner, J., Vullings, C., Madelain, L. \& van Beers, R. J. Prediction and final temporal errors are used for trial-to-trial motor corrections. Sci Rep 9, 19230 (2019).

21. Avraham, G., Sulimani, E., Mussa-Ivaldi, F. A. \& Nisky, I. Effects of visuomotor delays on the control of movement and on perceptual localization in the presence and absence of visual targets. Journal of Neurophysiology 122, 2259-2271 (2019).

22. Rohde, M. \& Ernst, M. O. Time, agency, and sensory feedback delays during action. Current Opinion in Behavioral Sciences 8, 193-199 (2016).

23. Krakauer, J. W., Hadjiosif, A. M., Xu, J., Wong, A. L. \& Haith, A. M. Motor learning. Comprehensive Physiology 9, 613-663 (2019).

24. Panico, F., Rossetti, Y. \& Trojano, L. On the mechanisms underlying Prism Adaptation: A review of neuro-imaging and neuro-stimulation studies. Cortex 123, 57-71 (2020).

25. Wei, K. \& Körding, K. Relevance of error: What drives motor adaptation? Journal of Neurophysiology 101, 655-664 (2009).

26. Wolpert, D., Ghahramani, Z. \& Jordan, M. An internal model for sensorimotor integration. Science 269, 1880-1882 (1995).

27. Diedrichsen, J. Optimal task-dependent changes of bimanual feedback control and adaptation. Current Biology 17, 1675-1679 (2007). 
28. Cousineau, D. Varieties of confidence intervals. ACP 13, 140-155 (2017).

29. Morey, R. D. Confidence intervals from normalized data: A correction to Cousineau (2005). TQMP 4, 61-64 (2008).

30. Grondin, S. About the (non)scalar property for time perception. in Neurobiology of Interval Timing (eds. Merchant, H. \& de Lafuente, V.) 17-32 (Springer, 2014). doi:10.1007/978-14939-1782-2_2.

31. Miyamoto, Y. R., Wang, S. \& Smith, M. A. Implicit adaptation compensates for erratic explicit strategy in human motor learning. Nat Neurosci 23, 443-455 (2020).

32. Tsay, J. S., Parvin, D. E. \& Ivry, R. B. Continuous reports of sensed hand position during sensorimotor adaptation. Journal of Neurophysiology 124, 1122-1130 (2020).

33. Taylor, J. A., Krakauer, J. W. \& Ivry, R. B. Explicit and implicit contributions to learning in a sensorimotor adaptation task. Journal of Neuroscience 34, 3023-3032 (2014).

34. McDougle, S. D., Ivry, R. B. \& Taylor, J. A. Taking aim at the cognitive side of learning in sensorimotor adaptation tasks. Trends in Cognitive Sciences 20, 535-544 (2016).

35. Morehead, J. R., Taylor, J. A., Parvin, D. E. \& Ivry, R. B. Characteristics of Implicit Sensorimotor Adaptation Revealed by Task-irrelevant Clamped Feedback. Journal of Cognitive Neuroscience 29, 1061-1074 (2017).

36. Taylor, J. A. \& Ivry, R. B. The role of strategies in motor learning. Annals of the New York Academy of Sciences 1251, 1-12 (2012).

37. Albert, S. T. et al. An implicit memory of errors limits human sensorimotor adaptation. Nat Hum Behav 1-15 (2021) doi:10.1038/s41562-020-01036-X.

38. Redding, G. M. \& Wallace, B. Calibration and alignment are separable: Evidence from prism adaptation. Journal of Motor Behavior 33, 401-412 (2001).

39. Burr, D., Tozzi, A. \& Morrone, M. C. Neural mechanisms for timing visual events are spatially selective in real-world coordinates. Nat Neurosci 10, 423-425 (2007).

40. Fornaciai, M., Arrighi, R. \& Burr, D. C. Adaptation-induced compression of event time occurs only for translational motion. Sci Rep 6, 23341 (2016).

41. Frassinetti, F., Magnani, B. \& Oliveri, M. Prismatic Lenses Shift Time Perception. Psychol Sci 20, 949-954 (2009).

42. Johnston, A., Arnold, D. H. \& Nishida, S. Spatially localized distortions of event time. Current Biology 16, 472-479 (2006).

43. Helmuth, L. L. \& Ivry, R. B. When two hands are better than one: Reduced timing variability during bimanual movements. Journal of Experimental Psychology: Human Perception and Performance 22, 278-293 (1996).

44. Gamache, P.-L. \& Grondin, S. Sensory-specific clock components and memory mechanisms: Investigation with parallel timing. European Journal of Neuroscience 31, 1908-1914 (2010).

45. Niehorster, D. C., Li, L. \& Lappe, M. The accuracy and precision of position and orientation tracking in the HTC Vive Virtual Reality system for scientific research. $i$-Perception $\mathbf{8}$, 204166951770820 (2017).

46. Verdelet, G. et al. Assessing spatial and temporal reliability of the Vive System as a tool for naturalistic behavioural research. in 2019 International Conference on 3D Immersion (IC3D) 1-8 (IEEE, 2019). doi:10.1109/IC3D48390.2019.8975994. 
47. Bates, D., Mächler, M., Bolker, B. \& Walker, S. Fitting Linear Mixed-Effects Models using lme4. J. Stat. Soft. 67, (2015).

48. Kuznetsova, A., Brockhoff, P. B. \& Christensen, R. H. B. ImerTest Package: Tests in Linear Mixed Effects Models. J. Stat. Soft. 82, (2017).

49. R Core Team. $R$ : A language environment for statistical computing. (R Foundation for Statistical Computing, 2017).

50. Morey, R. D. \& Rouder, J. N. BayesFactor: Computation of Bayes factors for common designs. (2015).

51. Maaß, S. C. \& Van Rijn, H. 1-s productions: A validation of an efficient measure of clock variability. Front. Hum. Neurosci. 12, 519 (2018).

52. Leys, C., Ley, C., Klein, O., Bernard, P. \& Licata, L. Detecting outliers: Do not use standard deviation around the mean, use absolute deviation around the median. Journal of Experimental Social Psychology 49, 764-766 (2013).

\section{Author Contributions}

NS participated in the design and data collection of Experiments 2-4, discussed the data, carried out data analysis and drafted the manuscript; TK participated in the design of Experiment 1-2, programmed Experiment 1-2, carried out data collection for Experiment 1 and gave feedback on the manuscript; MW adapted the code for Experiment 3, gave technical assistance and gave feedback on the manuscript; EZ participated in the design of the experiments, discussed the data and contributed to the writing of the manuscript. All authors gave final approval for publication. 